(C) 2015 IEEE. Personal use of this material is permitted. Permission from IEEE must be obtained for all other uses, in any current or future media, including reprinting/republishing this material for advertising or promotional purposes, creating new collective works, for resale or redistribution to servers or lists, or reuse of any copyrighted component of this work in other works. 


\section{Digital system for monitoring volcanic seismicity}

\author{
Normandino Carreras, Spartacus \\ Gomàriz, Antoni Mànuel \\ SARTI Research Group. Electronics \\ Dept. \\ Universitat Politécnica de Catalunya \\ (UPC). \\ Rambla Exposició 24, 08800, Vilanova \\ i la Geltrú. Barcelona. Spain \\ www.cdsarti.org
}

\author{
Angel David Moure, Pedro Antonio \\ Torres \\ Centro Geofísico de Canarias, \\ Instituto Geográfico Nacional (IGN) \\ C/ La Marina 20, $2^{\circ}$ Santa Cruz de \\ Tenerife, 38001, Spain
}

\author{
Ramón Ortiz \\ Institute IGEO, CSIC-UCM. J. \\ Gutierrez Abascal, 2, Madrid 28006, \\ Spain
}

\begin{abstract}
To control and preventing volcanic risk, are used different devices where many parameters are monitored that provide insight into the state of a volcano. This paper presents the design of system for monitoring the volcano seismicity. This system is composed of the module of acquisition data, the power module, the peripherals for communicate remotely and GPS to get time synchronization. This system is proposed to have a quick deployment in case of volcanic crisis, with a low cost of construction and maintenance. At the same time the equipment is built inside a watertight box, which allows installation of the system in adverse conditions and in remote locations with difficult access. The graph that can be seen in the results section shows a correct system operation.
\end{abstract}

Keywords—acquisition; seismic; volcano; low power; analog to digital; monitoring.

\section{INTRODUCTION}

The volcanoes, in the course of history, have become a subject of study due to the danger they pose to people living nearby. With the intention of preventing risk situations, monitoring the different parameters from volcano under study is carried out. Thereby can be reached on determine the state in which it is and how it can proceed with an action in response to the volcanic critical event [1].

A common fact in volcanology is that as changes in volcanic seismic activity is detected, additional tools must be installed to define better any increased activity. Similarly, it must be installed redundant equipment to improve system reliability to avoid the appearance of communication failures or any equipment. When volcanic activity begins to be more active, indicating the possibility of an eruption, then instruments are installed in inaccessible or highly threatened areas (called as "lost instrument”) to obtain more and better information of the data [2]. Due to the fact the instruments are designed to work for a limited time, because of the possibility of being lost, either of whether they are destroyed or even that their power source runs out, it is preferable that the measuring equipment are the lowest possible cost.

It can be found on the market a wide variety of equipment to study volcanoes through its seismology. Some digitizers equipment used in seismic acquisition are Taurus of Nanometrics [3] or CMG-DM24 of Güralp [4]. In both cases they may acquire up to 24-bits of resolution, with 3 channels at 100 samples per second, and that with remote data communication. Regarding the sensor used, we can find for example the 3Dlite of Lennartz [5] which working at $1 \mathrm{~Hz}$, or CMG-6T of Güralp that working to 30s of frequency. Both are active sensors that require external power and are composed of three axes.

The immense majority of them feature broadband geophones, designed for the acquisition of all sorts of earthquakes, although, its frequency range allows acquiring the signals produced by a volcano [6]. The problem of these devices is that they are bulky, expensive and geared primarily to measure signals triggered by earthquakes (being equipment intended for other purposes and exploited in volcanic). For example, the combination between digitizer and sensor from Güralp manufacturer exceeds the 10,000 euros, and, in case of Nanometrics, are even more expensive.

The next problem that emerges from commercial equipment lies in the autonomy, that generally have a high level of energy consumption, and if it is added to the necessity of having communication with a possible management center, these habitual communications, greatly aggravate the problem. For example, in both cases its power consumption is greater than $1 \mathrm{~W}$.

This article presents a design of a specific volcanic seismic acquisition system, with low power consumption, that enables rapid deployment in the event of volcanic crisis if occurs, and really cheap [7]. A device compact and with great autonomy, which for its size and weight allows easy installation, without maintenance. The equipment meets the following specifications:

- Low cost of purchase and maintenance.

- Low power consumption for long running periods.

- Use RF for remote communication.

- 3 channels of 24 bits.

- Sampling of 50 or 100 Sps (Samples per second)

- Lightweight and compact for transport and installation. 


\section{HARDWARE DESCRIPTION}

The system is divided in different modules. That allows the modification of parts of the system without changing it completely. The different parts are: the supply module, the overall management module, the communication peripherals, the sensors and outer box.

\section{A. Supply module}

The supply module is compound by Li-ion batteries assembled in an array of one single cell of $3.7 \mathrm{~V}$. The space where are located the batteries allows to incorporate twice of batteries to further increase the autonomy of the system. At the same time, an external connector exists to allow the connection of an external power, a fact that may further increase the autonomy.

In Figure 1, the supply board allows reduce the battery voltage to the different system voltage. This board is divided into 4 separate converters type MAX1684 of Texas Instruments, two for positive and negative voltage of $2.5 \mathrm{~V}$ and two voltages of $3.3 \mathrm{~V}$. In the communication part, the same voltage of $3.3 \mathrm{~V}$ is used, but its generation is composed of an independent converter from the system, in order to isolate the noise that might be transmitted through the power measures.

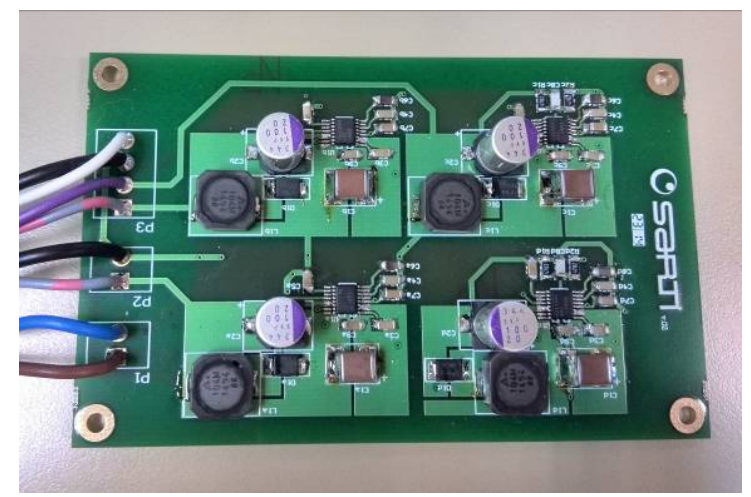

Fig. 1. Supply board.

\section{B. Overall Management module}

The overall management module mainly consists of a microprocessor MSP430F5438A of Texas Instruments, a low power chip which allows working until $25 \mathrm{MHz}$, enough to work and permit future additional functionalities, such as making a pre-processing of data before sending or storing. It has also three analog-digital converters ADS1246 of Texas Instruments with low power consumption, a SD storage memory, and a conditioning circuit for input sensors configured for three geophones kind SM-6 [7].

To facilitate commissioning of the equipment, this core module incorporates a blue LED, to indicate the state in which the system is and not lead to errors during the boot.

In Figure 2, it is presented the overall management module which has been designed and manufactured in PCB.

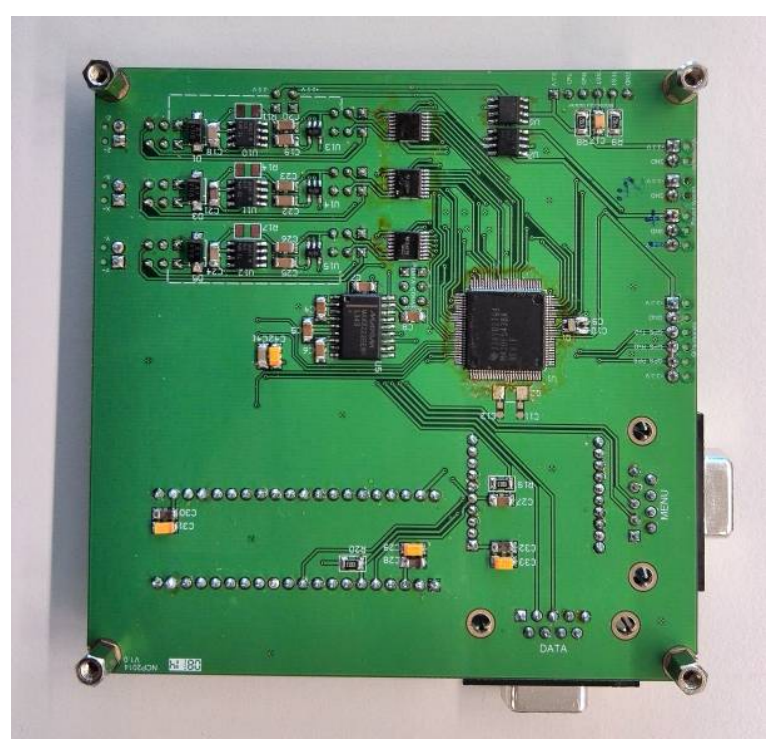

Fig. 2. Microprocessor board.

\section{Peripherals communication}

The system allows send data remotely, for this reason it incorporates a module from Lynx and a XBee module, which according to the manufacturer may have a coverage of $40 \mathrm{Km}$. At the same time, it offers the possibility of connect any communication equipment different those mentioned above, provided that the new module communicate via RS232. These three possibilities can only be used one at a time.

For communication between the user and the computer, there is a DB9 connector on the inside of the box through a serial reading application, using as example Hyperterminal, it is possible to interact and configure.

Among the peripherals, a GPS for time synchronization of the internal system clock is incorporated. The GPS used is the FGPMMOPA6H of GlobalTop Technology. It is important to note that GPS incorporates an internal antenna, which in case of outdoor installation, requires no additional antenna. The connection of an external antenna is thought for those cases in which the system is installed in a gallery or when reception is very limited and is necessary to install the antenna at a different point to receive more coverage.

\section{Sensors}

In the case of the sensors, the system has three geophones installed inside, nevertheless, it is possible to externally connect other sensors with their own conditioner circuit, or just the same but with the installation on the outside of the box [8].

The volcanic seismic acquisition requires the use of sensors working at frequency of about $1 \mathrm{~Hz}$, but they are expensive. To reduce costs and make the sensors more economically competitive, it has been facilitated the use of sensors of $4.5 \mathrm{~Hz}$ as SM6, which is cheaper and that, thanks to the action of a conditioning circuit, is achieved to shift the 
frequency work up to $1 \mathrm{~Hz}$. The ability to connect other kind of sensors in the system input, always within the range of acquisition, enables the use of the equipment in other working conditions, thus the system is much more flexible. Figure 3 shows the placement of three sensors inside a support structure constructed to be mounted in the system box.

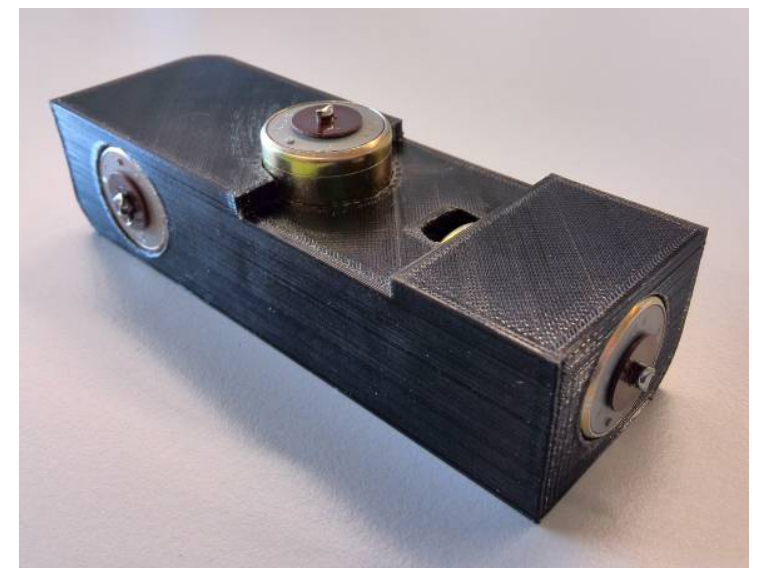

Fig. 3. Internal geophones placed on the three axes.

\section{E. External box}

The system is mounted in a sealed IP67 case with an external dimensions of $27 \times 24.6$ x $12.4 \mathrm{~cm}$ (see Figure 4), in order to withstand the weather and the adverse effects that can be found in the deployment area, as might be heavy rain, wind, frost, high humidity in the environment, etc. This box is equipped with four plug \& socket connections on the outside which allow the connection with the other elements of the system.

The first connector has 4-pin and allows connection of power externally or battery charging. The second connector composed of 7-pin, allows connection of sensors externally to the acquisition box. The last two connectors are designed for communications connections, one for GPS antenna and one for the RF antenna.

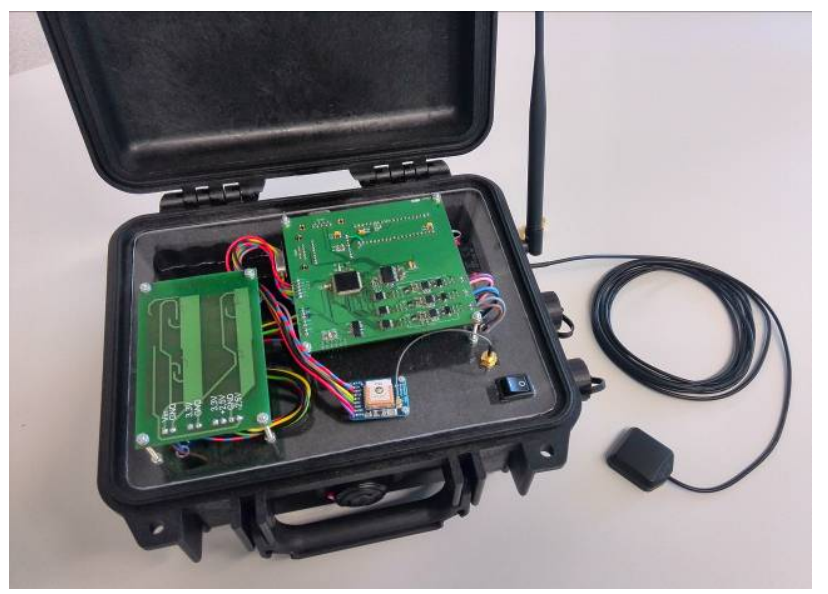

Fig. 4. Acquisition system.

\section{SYSTEM CONFIGURATION AND CHARACTERISTICS}

The unit has a setup menu that it let adjust the operating characteristics as required. Among those available parameters to set can be found: sampling, the number of active channels, data storage, and use of GPS.

The system is made up of three 24-bits channels and its sampling frequency can be set to 50 or 100 samples per second. There are three options for channel activation ranging from one to three if required. It will be 1 to 3 according to the selected channels respecting the order of their number (for example, for 2 channels will be active the channel one and two).

Once the data have been acquired, this data have three options of being treated. There are three possible configurations, only in memory storage (SD card), sending data only remotely, or the ability to record data in memory (SD) and remote delivery simultaneously.

The GPS can be configured so that the clock is synchronized every hour or every day, to work continuously, or to disable it.

When starting the system, the LED stays on indicating that it is waiting for the user to configure. After 10 seconds, if the user has not interacted with the system to configure anything, it will go into acquisition mode and the LED will blink at a rate of one second. If the user decides to stop the acquisition, entering the ending command through the menu connector, the acquisition will be finalized and the LED till turn off.

The structure given to the stored data, it is important that it is well defined so that the results can be studied better without losing information and for ensure compatibility when making comparisons between different stations. During storage or sending data, it is included a header with the information of the content that follows it. The information is stored or transmitted each second and is structured in the format of the following table I.

In information frame which is seen in table I, the first four bytes are the identifier string, following by the time data in Unix epoch format on bytes 4 to 7 . The byte number 8 inform about the source of data time, ' 1 ' indicates that the time data source correspond to the GPS, and a ' 0 ' to the RTC. Then it finds the information of the name station, which are composed of the bytes 9 to 12; a couple of bytes 13 and 14 for a counter that is incremented on each package; and finally the information on how the data are structured to be send: number of bits (byte 15), number of channels (byte 16) and number of samples per second (byte 17).

TABLE I. Header Structure Data

\begin{tabular}{|l|c|l|}
\hline \multicolumn{1}{|c|}{ index } & example & \multicolumn{1}{c|}{ description } \\
\hline Byte 0-3 & \$DAT & Data frame ID. \\
\hline Byte 4-7 & 1392800073 & Time in epoch format. \\
\hline Byte 8 & 1 & Time from GPS or inner. \\
\hline Byte 9-12 & SAR1 & Station name. \\
\hline
\end{tabular}




\begin{tabular}{|l|c|l|}
\hline Byte 13-14 & 05 & Frame number counter. \\
\hline Byte 15 & 24 & Number of bits by data. \\
\hline Byte 16 & 3 & Number of active channels. \\
\hline Byte 17 & 100 & Number of Samples per second. \\
\hline
\end{tabular}

The SD memory card which is working the device is class 4 , and it is accepting cards up to 32 GB of capacity. The format in which it is structured internally conforms to the FAT32 file system, and it has a folder structure for easy navigation between data. Each file that is created is only composed of the initial frame of each data packet and the acquired data itself. In normal operation with three channels, each hour the computer stores a file up to $3.3 \mathrm{MB}$, so with a 32GB memory can store data up to a year without the need to empty or delete files.

In the root folder appears first of all a folder with the name of the station, which is also set in the frame of data discussed above. Within this first folder is divided according to the date of acquisition of the data, being the first folder the year, and within that, a second folder with the month of acquisition. Ultimately, there are the files, in whose name includes information of the month (MM), day (DD) and the hour (HH) when it was initiated, followed by the raw extension. Thus, the data tree remains in the following way:

\section{/station_name/year/month/MMDDHH.raw}

\section{EXPERIMENTAL RESULTS}

Because of the different tests that have been conducted in the laboratory, it was possible to verify proper operation of each of the parts that make up the system, as well as the correct flow of data acquired from the input to the system, up to storage or sending. Thanks to the consumption essay it has confirmed that, as a result, it works with very low power consumption and that the power system behaves correctly. As a relevant data, it is remarkable that the system may be working (with RF-shutdown mode) consuming only about $150 \mathrm{~mW}$, a fact that allows long periods of operation of the system.

In the part dedicated to the file system, it has been possible to observe how they have been created different files within the folder structure established without posing any problem when it is creating new folders or either when creating new files. Another interesting point to be noted is the proper functioning in the generation of the frame that is used both in the storage of the data in the SD memory, as the remote transmission of those data.

For the realization of the first field test has been carried out of the system deployment on the El Hierro island (strategic area known for its seismic and volcanic instability) for a period of three consecutive days, thus confirming the correct operation, in real conditions and situations, of the system. In Figure 5, it is possible to appreciate a signal obtained during the acquisition period in El Hierro. In figure 5(a), there is the signal obtained with a Taurus of Nanometrics, the equipment as reference, with a 3Dlite sensor of Lennartz. On bottom of

that (figure 5(b)), the signal that is represented is the system under study.
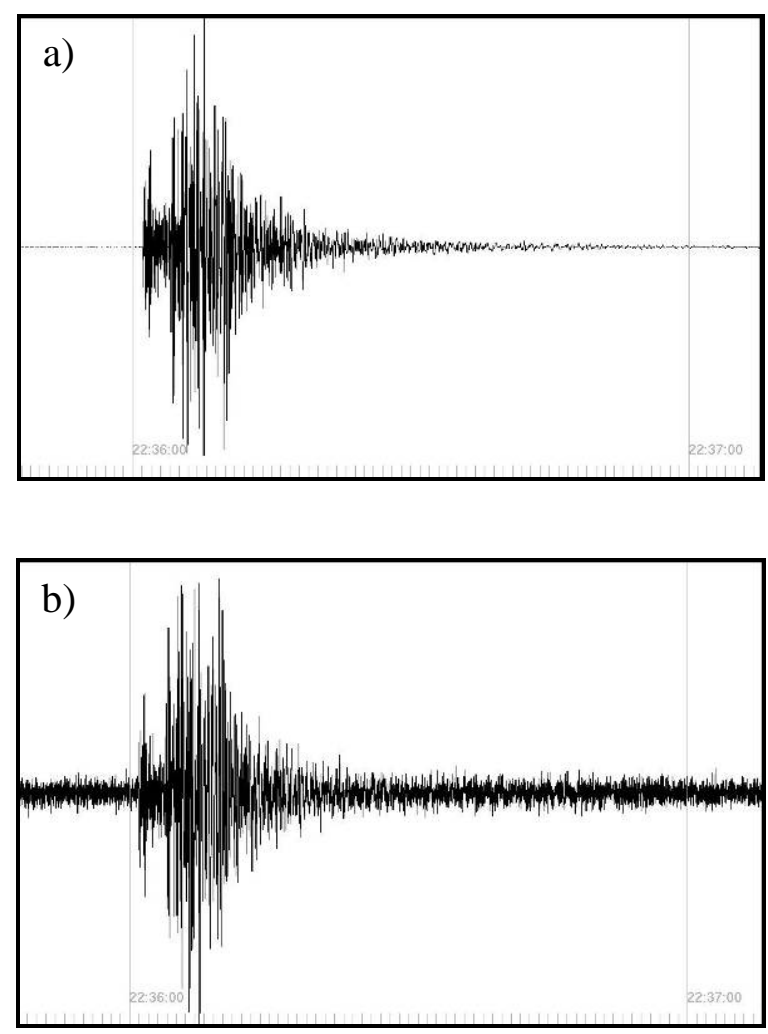

Fig. 5. Event obtained in El Hierro in the Canary Islands, with $3.8^{\circ}$ of magnitude, on 3rd September 2014. (a) Signal obtained by equipment as reference. (b) Signal obtained by equipment under study.

When comparing these figures obtained from different systems, it can say that event of $3.8^{\circ}$ of magnitude was registered correctly by the equipment developed. However, is possible to appreciate that commercial system has a better signal/noise ratio.

With the results of this first field test, it is calculated that acquisition noise exceeds 10,000 counts. In the laboratory it was possible to identify the noise coming from the ADC circuitry, in particular a power supply. The problem was solved by replacing the component in charge to generate ADC negative reference signal, for another one with better SNR. This resulted in a level of noise in the acquisition up to 50 counts.

After solve some problem with components, it's improved this ratio. And a second field test in Las Cañadas of Tenerife, in the same Canary Island. The period of these tests is performed during the second week of December 2014. In figure 6 can be seen the acquisition obtained on 13th December. 


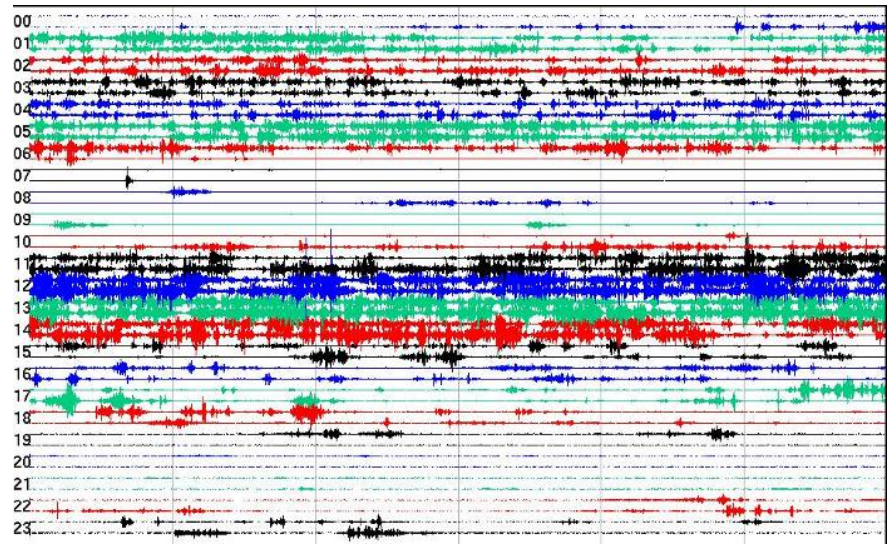

Fig. 6. Signals obtained in Las Cañadas of Tenerife in the Canary Islands, on 13th December 2014.

In that chart of figure 6 , it can be seen some signals of noise. But if this data is compared with seismic catalogue from IGN (National Geographic Institute), it can be found an event of $1.7^{\circ}$ of magnitude at 7:33 UTC (figure 7(b)). In figure 7(a), it is showing the signal from DM24 of Güralp, the equipment as reference, along with a CMG-6T, sensor of Güralp, too. At the end, it can be find the system under study.
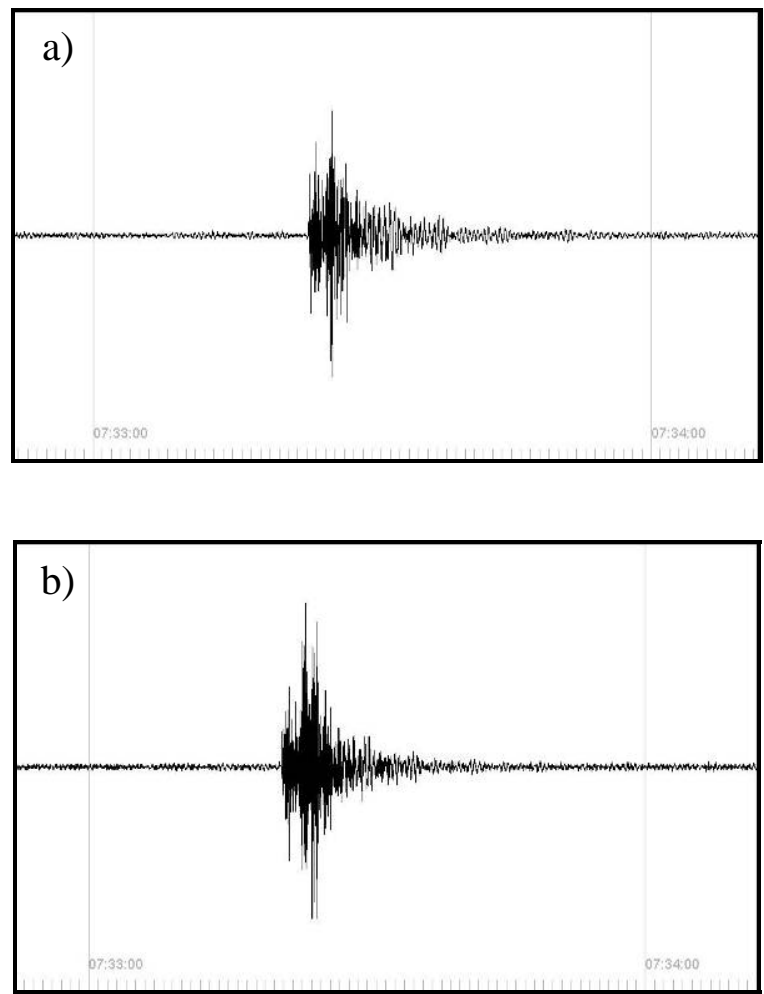

Fig. 7. Event obtained in Las Cañadas of Tenerife in the Canary Islands, with $1.7^{\circ}$ of magnitude, on 13th December 2014. (a) Signal from reference system. (b) Signal from system under study.

In figure 7 , can observe an event of $1.7^{\circ}$ of magnitude, an event lower compared to the last picture (figure 5), and in this case the system developed has registered it perfectly. Also, be seen a better signal/noise ratio if compared figure 7(b) with figure 5(b), although it can still improve.

\section{CONCLUSIONS}

It has been obtained a seismic acquisition system for volcano monitoring, with possibility to use different methods of communication, with GPS synchronization with indoor or outdoor antenna, data storage removable SD memory, external connectors for the possibility of connect an external power supply, external sensors and to connect the RF antenna. All of that makes it flexible.

The fact of incorporating a conditioner circuit in the system to reduce the frequency of $4.5 \mathrm{~Hz}$ to a working frequency of $1 \mathrm{~Hz}$, like the case of SM-6 sensor, it provides a significant economic reduction when this equipment is made, and flexibility in the use of different sensors.

With components that have been selected it has been achieved that the equipment to be low cost. At the same time, thanks to the low power of the system allow less maintenance and therefore less maintenance cost.

The system that has been created has low power consumption and it has built compactly. Its design allows the deployment of the equipment in inaccessible places or too threatened by the effect of a volcano, without making a large investment.

In the results section, it has been mentioned that the system has been improved in the signal/noise ratio, but this field can still improve, and we are still working on it. One of this improvements in which we are working on, consists in to amend the system signal filtering in the analog part to ameliorate the obtained results. Another focus of improvement in which we are working, is the possibility to incorporate an algorithm to processing data before the sending. It aims to perform a reduction of data send and a reduction of power consumption by the communications module. This would allow increasing the working time.

\section{Acknowledgment}

This work was supported by the Spanish Ministry of Economy and Competitiveness under the research project: "Peligro Volcánico y Evaluación del Riesgo en Tenerife" (CGL2011-28682-C02-02)

\section{References}

[1] Ramón Ortiz, Alicia García, Mar Astiz "Instrumentación en volcanología”. Editor Excmo. Cabildo Insular de Lanzarote. Octubre 2001. ISBN 84-97021-84-0.

[2] J. M. Ibáñez, E. Carmona. "Sismicidad volcánica”. Instituto de Geofísica. Universidad de Granada. Campus de Cartuja s/n. 18071 Granada. $\quad$ Retrieved January 2015 http://www.uclm.es/profesorado/egcardenas/SISMICIDA D_VOLCANICA[1].pdf

[3] http://www.nanometrics.ca/seismology/products/ Retrieved January 9, 2015

[4] http://www.guralp.com/products Retrieved January 9, 2015 
[5] http://www.lennartz-electronic.de/ Retrieved January 9, 2015

[6] José M. azañón Hernandez, Antonio Azor Pérez, Francisco M. Alonso Chaves, Miguel Orozco Fernández. “Geología Física”. Ed. Paraninfo. 2002. ISBN 84-9732-021-2

[7] N. Carreras, A. Mànuel, S. Gomariz. "Design of seismic acquisition system for volcanology” IMEKO TC-4 2013, Symposium Measurements of Electrical Quantities. 2013 July 18-19, Barcelona (Spain) ISBN 978-84-616-5438-3
[8] Normandino Carreras, Spartacus Gomariz, and Antoni Manuel. "Small instrument to volcanic seismic signals”. EGU General Assembly 2014, 27 April - 02 May 2014, Vienna (Austria) 